\title{
Ascending aorta: the use of a physician investigational device exemption
}

\author{
Ali Khoynezhad, Tiffany Worthington, Rodney White \\ MemorialCare Heart and Vascular Institute, Long Beach, CA, USA \\ Correspondence to: Ali Khoynezhad. Department of Cardiovascular Surgery, MemorialCare Heart and Vascular Institute, 2801 Atlantic Avenue, Long \\ Beach, CA 90806, USA. Email: akhoynezhad@memorialcare.org.
}

Submitted Mar 06, 2021. Accepted for publication Oct 04, 2021.

doi: 10.21037/acs-2021-taes-32

View this article at: https://dx.doi.org/10.21037/acs-2021-taes-32

\section{Introduction}

Since the initial reports in 1986, endovascular approaches have revolutionized treatment of aortic disease from abdominal aorta to the aortic arch. The ascending aorta (AA) remains the final frontier in endovascular aortic surgery. Initial reports of off-label use of stent grafts in the AA were published in 2000, however to date, there are still no commercially available stent grafts approved by the Food and Drug Administration (FDA) for use in the AA (1). Continued experience over the past twenty-one years includes numerous case series and case reports with a variety of devices and approaches. Meta-analysis of these case series and reports, identified a technical success rate as high as $95.5 \%$ with early mortality as low as $2.9 \%(2,3)$.

There are a variety of challenges unique to the AA when compared to more distal regions of the aorta. Despite being the only branchless segment of the aorta, significant obstacles in the landing zones of the AA exist, including the coronary ostia and the aortic valve proximally, as well as the innominate artery in the distal landing zone. Hemodynamic forces and variation in aortic diameter between cardiac cycles is more significant in the AA, making positioning and sizing challenging (3). To accommodate the anatomic challenges unique to the AA, stent grafts must be shorter in length and larger in diameter than those used in the distal aorta. Increased curvature in the AA also requires conformability to allow for coaxial deployment.

Through the investigational device exemption (IDE) process with the FDA, clinical trials can be performed to evaluate the safety and efficacy of AA stent grafts for potential use in a larger population. Currently two FDA approved physician sponsored (PS) IDE trials are enrolling subjects with ascending aortic disease for treatment with an endovascular stent (EVOLVE Aorta - Massachusetts General, Boston, MA and Medtronic Valiant PS-IDE MemorialCare Long Beach Medical Center, Long Beach, CA and Baylor Research Institute, Plano TX, USA).

\section{IDE application process}

Aortic stent grafts are classified as significant-risk devices by the FDA. This classification is based on the determination by the FDA that the devices pose a serious risk to the health, safety or welfare of a subject (4). Any clinical trials involving a significant-risk device requires IDE approval by the FDA prior to proceeding. Applications for IDE can be industry-sponsored or physician-sponsored (PS-IDE). The criteria for scientific justification and conduct of the study are the same regardless of sponsor, however if an industry sponsored IDE is sought, the application will include items such as training protocols and device marketing information. In PS research, the pursuit and advancement of scientific knowledge of the device is the primary objective. The study is built to test a hypothesis, and industry involvement is often limited to following data for serious adverse events and reviewing documentation. With industry sponsored research, bias can exist towards protecting shareholder interests, which may affect how outcomes are reported. Ultimately, the principle investigator is conducting the clinical trial in PS-IDE, whilst device companies are in charge for standard IDEs. 
The FDA outlines medical device law and medical regulations as interpreted in the Federal Register that must be considered when seeking investigational approval. During the initial IDE application process, a pre-submission can be made to the FDA allowing for feedback and recommendations prior to submission of the final application. The IDE application for FDA approval includes a complete investigational plan, description of prior investigations, description of the manufacturing process for the device being investigated, details pertaining to labeling, informed consent and data collection (5). Upon submission of the IDE application, the FDA will generate an IDE number and reply within thirty days regarding whether the application has been awarded approval, approval with conditions or disapproved. If disapproved, the sponsor can initiate an appeal through a regulatory hearing (5).

Along with PS-IDE approval, investigators must seek Institutional Review Board (IRB) approval through the institution where the study will take place. During the course of the clinical trial, details on conduct of the study including patient consent, reimbursement and data collection must be reported to both the IRB and FDA.

Once these approvals have been obtained, a request and approval through Center for Medicare and Medicaid Services (CMS) should be submitted to secure procedural and device reimbursement. The Medicare Prescription Drug, Improvement and Modernization Act (MMA) of 2003 allows for Medicare payment of costs associated with IDE studies. During the IDE approval process with the FDA, the investigational device is categorized as A (experimental) or B (nonexperimental/investigational) (6). This determination is forwarded to CMS and the study sponsor simultaneously during the IDE process. Devices are classified as Category $\mathrm{A}$ if there are unresolved initial questions pertaining to the safety and efficacy of the device. Category B devices are those that are known to be safe and effective based on prior investigations and other manufacturers have obtained FDA premarket approval or clearance for that device type (5). If the IDE is considered Category A, the routine care items and services furnished in the study will be reimbursed, but the device will not. In Category B investigations, the device will also be approved for reimbursement (6). In general, aortic stent grafts are classified as Category B.

\section{Acknowledgments}

Funding: None.

\section{Footnote}

Conflicts of Interest: The authors have no conflicts of interest to declare

Open Access Statement: This is an Open Access article distributed in accordance with the Creative Commons Attribution-NonCommercial-NoDerivs 4.0 International License (CC BY-NC-ND 4.0), which permits the noncommercial replication and distribution of the article with the strict proviso that no changes or edits are made and the original work is properly cited (including links to both the formal publication through the relevant DOI and the license). See: https://creativecommons.org/licenses/by-nc-nd/4.0/.

\section{References}

1. Dorros G, Dorros AM, Planton S, et al. Transseptal guidewire stabilization facilitates stent-graft deployment for persistent proximal ascending aortic dissection. J Endovasc Ther 2000;7:506-12.

2. Baikoussis NG, Antonopoulos CN, Papakonstantinou NA, et al. Endovascular stent grafting for ascending aorta diseases. J Vasc Surg 2017;66:1587-601.

3. Khoynezhad A, Donayre CE, Walot I, et al. Feasibility of endovascular repair of ascending aortic pathologies as part of an FDA-approved physician-sponsored investigational device exemption. J Vasc Surg 2016;63:1483-95.

4. U.S. Food and Drug Administration. CFR - Code of Federal Regulations. 21 CFR 812.20 Available online: https://www.accessdata.fda.gov/scripts/cdrh/cfdocs/cfCFR/ CFRSearch.cfm?FR=812.20 Accessed March 1, 2021.

5. U.S. Food and Drug Administration. Investigational Device Exemption. Available online: https://www.fda. gov/medical-devices/how-study-and-market-your-device/ investigational-device-exemption-ide. Accessed March 1, 2021.

6. U.S. Food and Drug Administration. CFR - Code of Federal Regulations. 42 CFR $\$ 405$ Subpart B. Available online: https://www.cms.gov/Medicare/Coverage/IDE. Accessed March 1, 2021.

Cite this article as: Khoynezhad A, Worthington T, White R. Ascending aorta: the use of a physician investigational device exemption. Ann Cardiothorac Surg 2022;11(1):48-49. doi 10.21037/acs-2021-taes-32 